\title{
Resistencia a antibióticos y epidemiología molecular de Shigella spp. en el nordeste argentino
}

\author{
Luis A. Merino, ${ }^{1}$ Gabriela E. Hreñuk, ${ }^{1}$ María C. Ronconi ${ }^{1}$ y José M. Alonso ${ }^{1}$
}

Forma de citar

Merino LA, Hreñuk GE, Ronconi MC, Alonso JM. Resistencia a antibióticos y epidemiología molecular de Shigella spp. en el nordeste argentino. Rev Panam Salud Publica. 2004;15(4):219-24.

RESUMEN Objetivos. Evaluar la resistencia a antibióticos de cepas de Shigella spp. aisladas de muestras de heces en el nordeste argentino y caracterizarlas desde el punto de vista de su epidemiología molecular.

Métodos. Se estudiaron 132 aislamientos de Shigella spp. obtenidos de las heces de igual número de pacientes con diarrea que asistieron a diferentes laboratorios privados y estatales de las provincias del Chaco y Corrientes, Argentina, durante el período de 1998 a 2002. Cada cepa se caracterizó según su serotipo, su resistencia a 13 antibióticos individuales o combinados y su sensibilidad a las piocinas. A 52 cepas seleccionadas en función de sus perfiles de susceptibilidad antimicrobiana se les determinaron la dotación plasmídica mediante lisis alcalina y las secuencias repetitivas palindrómicas extragénicas mediante la amplificación de segmentos repetitivos de $A D N$ con la reacción en cadena de la polimerasa (REP-RCP). Se aplicó la prueba de ji al cuadrado para comparar proporciones. El nivel de significación estadística fue de 0,05. Resultados. Shigella flexneri fue la especie más frecuente (78\%), seguida de S. sonnei (22\%). En general, la resistencia de S. flexneri a los antibióticos estudiados fue mayor que la de S. sonnei y esta diferencia fue estadísticamente significativa $(\mathrm{P}<0,001)$ frente a ampicilina, tetraciclina, cloramfenicol y la combinación de ampicilina con sulbactama. Las cepas de S. flexneri también mostraron mayor multirresistencia que las de S. sonnei $(84,5 \%$ frente a $31,0 \% ; \mathrm{P}<0,001)$. Las cepas aisladas de S. flexneri pudieron agruparse según 5 piocinotipos, 3 perfiles plasmídicos y 5 patrones de secuencias repetitivas palindrómicas. Por su parte, las cepas de S. sonnei conformaron 3 piocinotipos, 2 perfiles plasmídicos y 3 patrones de secuencias repetitivas palindrómicas.

Conclusiones. Las especies de Shigella estudiadas mostraron una elevada resistencia a los antibióticos de uso más frecuente, por lo que se deben poner en marcha actividades de vigilancia a fin de detectar y controlar la aparición de nuevas cepas resistentes. La aplicación de técnicas de tipificación epidemiológica puede ayudar a conocer con mayor precisión la distribución y evolución de cepas de microorganismos resistentes circulantes.

Palabras clave Shigella, resistencia microbiana a las drogas, epidemiología molecular, Argentina.

Las diarreas agudas de origen infeccioso constituyen una de las causas más

\footnotetext{
Instituto de Medicina Regional, Universidad Nacional del Nordeste, Resistencia, Argentina. La correspondencia debe dirigirse a Luis A. Merino, Av. Maipú 1550 2A, Corrientes, Argentina. Correo electrónico: lmerino@arnet.com.ar
}

frecuentes de mortalidad infantil en los países en desarrollo, aunque muchas de ellas - como la shigelosis - pueden ser tratadas eficazmente con antibióticos (1). La sensibilidad de las especies del género Shigella a los antibióticos ha evolucionado en los últimos años de- bido a la fuerte presión selectiva impuesta por el uso irracional de estos medicamentos. Este fenómeno se ha reflejado en el aumento del número de enfermedades causadas por cepas resistentes, tanto en Argentina como en otros países $(1,2)$. 
La tipificación epidemiológica de las cepas bacterianas es un elemento importante para identificar los brotes infecciosos, determinar la fuente de la infección y reconocer las cepas particularmente virulentas (3). Entre las técnicas de tipificación más frecuentemente empleadas se encuentran la clasificación por biotipos, serotipos, antibiotipos y piocinotipos bacterianos; el análisis de plásmidos; y el estudio del ácido desoxirribonucleico (ADN) cromosómico mediante electroforesis en campo pulsátil (PFGE) o por amplificación de segmentos repetitivos de ADN mediante la reacción en cadena de la polimerasa (REP-RCP) (4).

El objetivo de este trabajo fue evaluar la resistencia a antibióticos de las cepas de Shigella spp. aisladas de muestras de heces en la región del nordeste argentino y caracterizarlas desde el punto de vista de su epidemiología molecular.

\section{MATERIALES Y MÉTODOS}

Se estudiaron 132 aislamientos de Shigella spp. obtenidos de heces de igual número de pacientes con diarrea que asistieron a diferentes laboratorios privados y estatales de las provincias del Chaco y Corrientes, Argentina, durante el período de 1998 a 2002. Las muestras se recolectaron por emisión espontánea en frascos estériles de boca ancha y se enviaron a los laboratorios en el transcurso de la siguiente hora para su procesamiento. Las muestras se analizaron macroscópicamente y al microscopio mediante montaje húmedo y se sembraron en agar con tripteína soya suplementado con $5 \%$ de sangre, agar eosina-azul de metileno y agar para Salmonella-Shigella. Las cepas identificadas preliminarmente como Shigella spp. mediante pruebas bioquímicas clásicas se inocularon en agar nutritivo al $7 \%$ y se enviaron al Instituto de Medicina Regional, en la ciudad de Resistencia, donde se confirmó su identificación mediante pruebas bioquímicas y serológicas y se efectuaron pruebas de sensibilidad a antibióticos. Los aislamientos se mantuvieron a $-20{ }^{\circ} \mathrm{C}$ en infusión de cerebro y corazón con glicerina al $15 \%$ hasta que se hicieron las pruebas de biología molecular.

Para la tipificación serológica de las cepas se utilizó la técnica de aglutinación en placa con antisueros comerciales contra los grupos de Shigella A, B, C y D (Sanofi Diagnostics Pasteur, Francia). Las cepas se enviaron posteriormente inoculadas en agar al 7\% al Instituto Nacional de Enfermedades Infecciosas Dr. Carlos G. Malbrán, de la ciudad de Buenos Aires, Argentina, donde se confirmó el grupo serológico y se determinó el serotipo según correspondiera, de acuerdo con lo establecido en el país.

La sensibilidad de las cepas se evaluó mediante la técnica de difusión en agar (5) con los siguientes antibióticos: $10 \mu \mathrm{g}$ de ampicilina (AMP); $10 \mu \mathrm{g}$ de ampicilina con $10 \mu \mathrm{g}$ de sulbactama (AMS); $10 \mu \mathrm{g}$ de cefalotina (CEF); 10 $\mu \mathrm{g}$ de gentamicina (GEN); $30 \mu \mathrm{g}$ de neomicina (NEO); $30 \mu \mathrm{g}$ de tetraciclina (TET); $30 \mu \mathrm{g}$ de cloranfenicol (CMP); $10 \mu \mathrm{g}$ de colistina (COL); $30 \mu \mathrm{g}$ de ácido nalidíxico (NAL); $5 \mu \mathrm{g}$ de ciprofloxacina (CIP); $100 \mu \mathrm{g}$ de furazolidona (FUR); $200 \mu \mathrm{g}$ de fosfomicina (FOS); y una mezcla de $1,25 \mu \mathrm{g}$ de trimetoprima con 23,75 $\mu \mathrm{g}$ de sulfametoxazol (TMS). Como control de las pruebas de sensibilidad se utilizó la cepa de Escherichia coli $\mathrm{ATCC}^{\circledR} 25922$.

Se determinó la sensibilidad de todos los aislamientos a bacteriocinas de Pseudomonas aeruginosa (piocinas) mediante el método de estrías cruzadas descrito por Wahba (6), utilizando cepas productoras pertenecientes a la colección de los autores (7).

Se analizó la dotación plasmídica de 52 cepas escogidas por ser multirresistentes o por compartir igual perfil de susceptibilidad, mediante la técnica de lisis alcalina descrita por Kado y Liu (8) y modificada por Nakamura y colaboradores (9). Se aisló el ADN plasmídico mediante electroforesis en gel de agarosa al $0,75 \%$ y se determinaron los pesos moleculares por comparación visual con los plásmidos presentes en las cepas de referencia de Escherichia coli V517 y 39R861.
Se amplificaron las secuencias repetitivas palindrómicas extragénicas de las cepas de igual serotipo que podían tener alguna relación epidemiológica por provenir de un mismo sitio y haber sido aisladas en fechas cercanas. Para ello se aplicó el método de REPPCR descrito por Gallardo y colaboradores (10) con el cebador REP3: 5'GCG CCG ICA TGC GGC ATT-3' (Invitrogen Corporation, California, EUA). Para visualizar mediante electroforesis los segmentos amplificados, se colocaron $20 \mu \mathrm{L}$ del producto final de cada reacción en geles de agarosa al $1,5 \%$ con $0,5 \mu \mathrm{g} / \mathrm{mL}$ de bromuro de etidio. Los segmentos se fotografiaron con películas Polaroid. Para la comparación de pesos moleculares se utilizó un marcador de 100 bp. Se emplearon controles negativos y controles positivos de amplificación de beta-globina.

Los datos se registraron y analizaron mediante el programa Epi Info (versión 6.04b). Se aplicó la prueba de ji al cuadrado para comparar proporciones y el nivel de significación estadística se fijó en 0,05.

\section{RESULTADOS}

De las 132 cepas de Shigella estudiadas, $29(22 \%)$ correspondieron a S. sonnei y $103(78 \%)$ a S. flexneri. De estas últimas, ocho cepas eran del serotipo 6, tres pertenecían al serotipo 3, una correspondía al serotipo 1 y las 91 cepas restantes pertenecían al serotipo 2 .

En general, la resistencia de $S$. flexneri a los antibióticos estudiados fue mayor que la de $S$. sonnei y esta diferencia fue estadísticamente significativa $(P<0,001)$ para los antibióticos AMP, AMS, TET y CMP (cuadro 1). Las cepas de $S$. flexneri también mostraron mayor multirresistencia que las de $S$. sonnei $(84,5 \%$ frente a $31,0 \%$; $P<$ 0,001 ) (cuadro 2).

Con respecto a los patrones de susceptibilidad obtenidos frente a la producción de piocinas por parte de las cepas de Pseudomonas aeruginosa, las cepas de $S$. flexneri se agruparon en 5 piocinotipos, mientras que las de $S$. sonnei conformaron 3 piocinotipos di- 
ferentes, independientemente del perfil de resistencia que presentaran a los antibióticos (cuadro 3).

Entre los aislamientos de S. sonnei seleccionados de acuerdo con sus perfiles de susceptibilidad antimicrobiana se observaron dos perfiles plasmídicos que solo diferían en un plásmido de 5,0 kb; entre las cepas multirresistentes de $S$. flexneri se encontraron tres perfiles que diferían entre sí por la presencia de uno o dos plásmidos (cuadro 4). Además, todas las cepas contenían un plásmido de aproximadamente $130 \mathrm{~kb}$. En la figura 1 se muestran los diferentes patrones plasmídicos observados.

En cuanto a la caracterización mediante la técnica de REP-PCR, las cepas de $S$. flexneri se agruparon en cinco perfiles según las bandas de $A D N$ que generaban (A, B, C, D y E), caracterizadas por una gran diversidad de tamaños, mientras que las cepas de $S$. sonnei se agruparon en tres perfiles ( $\mathrm{F}, \mathrm{G}$ y $\mathrm{H}$ ) con mayor homogeneidad en el tamaño de las bandas (figura 2).

En el cuadro 4 se resumen las características de las cepas estudiadas y la relación encontrada entre los perfiles más frecuentes de resistencia a antibióticos y los demás marcadores epidemiológicos.

\section{DISCUSIÓN}

Los resultados obtenidos en el presente estudio con cepas de Shigella aisladas de muestras clínicas confirman resultados previos que revelan una mayor prevalencia de $S$. flexneri, particularmente del serotipo 2, seguida de S. sonnei en Argentina $(2,11)$ y otros

CUADRO 1. Resistencia a antimicrobianos en cepas de Shigella aisladas en la región nordeste de Argentina, 1998-2002

\begin{tabular}{|c|c|c|c|c|c|}
\hline \multirow[b]{3}{*}{ Antibiótico } & \multirow{2}{*}{\multicolumn{2}{|c|}{$\begin{array}{c}\text { S. flexneri }(N=103) \\
\begin{array}{c}\text { Cepas } \\
\text { resistentes }\end{array} \\
\end{array}$}} & \multirow{2}{*}{\multicolumn{2}{|c|}{$\begin{array}{c}\text { S. sonnei }(N=29) \\
\text { Cepas } \\
\text { resistentes }\end{array}$}} & \multirow[b]{3}{*}{$P^{a}$} \\
\hline & & & & & \\
\hline & No. & $\%$ & No. & $\%$ & \\
\hline Ampicilina & 83 & 80,6 & 11 & 37,9 & $<0,001$ \\
\hline Tetraciclina & 66 & 64,1 & 2 & 6,9 & $<0,001$ \\
\hline Trimetoprima/sulfametoxazol & 61 & 59,2 & 18 & 62,1 & 0,78 \\
\hline Cloranfenicol & 57 & 55,3 & 2 & 6,9 & $<0,001$ \\
\hline Ampicilina/sulbactama & 47 & 45,6 & 2 & 6,9 & $<0,001$ \\
\hline Cefalotina & 17 & 16,5 & 2 & 6,9 & 0,19 \\
\hline Gentamicina & 7 & 6,8 & 0 & 0,0 & 0,17 \\
\hline Furazolidona & 7 & 6,8 & 0 & 0,0 & 0,17 \\
\hline Fosfomicina & 6 & 5,8 & 2 & 6,9 & 0,55 \\
\hline Colistina & 4 & 3,9 & 1 & 3,4 & 0,91 \\
\hline Ácido nalidíxico & 3 & 2,9 & 1 & 3,4 & 0,88 \\
\hline Neomicina & 2 & 1,9 & 0 & 0,0 & 0,60 \\
\hline Ciprofloxacina & 0 & 0,0 & 0 & 0,0 & Indefinido \\
\hline
\end{tabular}

${ }^{a}$ Nivel de significación de 0,05 .

países $(12,13)$, aunque algunos autores han encontrado un predominio de los aislamientos de $S$. sonnei $(14,15)$.

Entre los hallazgos del presente trabajo debe destacarse el elevado número de aislamientos resistentes a AMP y TMS. En los últimos años se ha observado el aumento de la resistencia frente a estos antibióticos en Argentina en comparación con 1995-1996 (16) y 2000 (11), lo cual limita el empleo de estos medicamentos antimicrobianos en el tratamiento de las diarreas producidas por Shigella. Esta tendencia también se ha observado en otros países (17).

Los niveles de resistencia a NAL son similares a los encontrados por otros autores $(1,12,18)$. Aunque no se detectaron aislamientos resistentes a CIP, la aparición de cepas resistentes a NAL debe servir de alerta, ya que estudios realizados en Salmonella spp. han demostrado que la resistencia a este último antibiótico puede indicar que ha disminuido la sensibilidad frente a las quinolonas fluoradas (19).

La alta proporción de multirresistencia encontrada en ambas especies de Shigella ya ha sido demostrada previamente por Vila y colaboradores en cepas aisladas de pacientes con diarrea del viajero (20), por Lima y colaboradores en Fortaleza, Brasil, (21) y por Boehme y colaboradores en Temuco, Chile (22). El hecho de que $S$. flexneri sea la especie encontrada con mayor frecuencia y la de mayor multirresistencia constituye un grave problema médico y sanitario, ya que se reducen las posibilidades terapéuticas, princi-

CUADRO 2. Distribución de las cepas de Shigella resistentes a más de un antimicrobiano (multirresistentes) según la especie y el número de antibióticos frente a los cuales presentaron resistencia. Región nordeste de Argentina, 1998-2002

\begin{tabular}{|c|c|c|c|c|c|c|c|c|c|c|}
\hline \multirow[b]{2}{*}{ Especie } & \multirow{2}{*}{$\begin{array}{l}\text { No. de } \\
\text { cepas } \\
\text { aisladas }\end{array}$} & \multicolumn{7}{|c|}{$\begin{array}{l}\text { Número de antibióticos frente a los } \\
\text { cuales hubo resistencia }\end{array}$} & \multicolumn{2}{|c|}{$\begin{array}{c}\text { Cepas } \\
\text { multirresistentes }\end{array}$} \\
\hline & & 2 & 3 & 4 & 5 & 6 & 7 & 8 & No. & $\%$ \\
\hline S. flexneri & 103 & 11 & 15 & 24 & 23 & 12 & 1 & 1 & 87 & 84,5 \\
\hline S. sonnei & 29 & 5 & 1 & 1 & 1 & 1 & 0 & 0 & 9 & 31,0 \\
\hline Total & 132 & 16 & 16 & 25 & 24 & 13 & 1 & 1 & 96 & 72,7 \\
\hline
\end{tabular}


CUADRO 3. Perfiles de susceptibilidad a piocinas (piocinotipos) obtenidos en las cepas de Shigella aisladas. Región nordeste de Argentina, 1998-2002

\begin{tabular}{ccccccc}
\hline & \multicolumn{5}{c}{ Cepas de Pseudomonas aeruginosa productoras de piocinas } \\
\cline { 2 - 7 } Piocinotipo & NF217 & NF220 & NF237 & NF249 & NF252 & NF257 \\
\hline I & S & R & S & S & S & S \\
II & S & S & R & S & S & R \\
III & S & S & S & R & R & R \\
IV & S & R & S & S & R & S \\
V & R & R & S & S & S & S \\
VI & R & S & R & S & S & R \\
VII & R & R & S & R & R & S \\
VIII & S & S & S & S & S & S \\
\hline
\end{tabular}

$\mathrm{S}=$ cepa sensible; $\mathrm{R}=$ cepa resistente.

CUADRO 4. Relación entre los perfiles de resistencia antimicrobiana más frecuentes en cepas de Shigella aisladas y otros marcadores epidemiológicos. Región nordeste de Argentina, 1998-2002

\begin{tabular}{|c|c|c|c|c|c|}
\hline \multirow[b]{2}{*}{ Especie } & \multicolumn{4}{|c|}{ Marcadores epidemiológicos } & \multirow[b]{2}{*}{$\begin{array}{l}\text { No. de } \\
\text { cepas }\end{array}$} \\
\hline & $\begin{array}{l}\text { Fenotipo de } \\
\text { resistencia }^{a}\end{array}$ & $\begin{array}{l}\text { Perfil plasmídico } \\
\text { (pesos aprox. } \\
\text { en kb) }\end{array}$ & Piocinotipob $^{b}$ & REP-PCR ${ }^{c}$ & \\
\hline \multirow[t]{10}{*}{ S. flexneri } & $\begin{array}{l}\text { AMPr } \text { AMS }^{r} \mathrm{TET}^{\mathrm{r}} \\
\mathrm{CMP}^{\mathrm{r}} \mathrm{TMS}^{\mathrm{r}}\end{array}$ & $130 ; 70 ; 5,0 ; 2,5$ & I & A & 6 \\
\hline & & & II & B & 5 \\
\hline & & & III & C & 4 \\
\hline & & & IV & D & 4 \\
\hline & $\begin{array}{l}\text { AMPr } \text { TETr }^{r} \text { CMPr } \\
\text { TMS }^{r}\end{array}$ & $130 ; 7,0 ; 2,5$ & I & A & 5 \\
\hline & & & IV & D & 4 \\
\hline & & & V & E & 2 \\
\hline & $\mathrm{AMP}^{r} \mathrm{TET}^{\mathrm{r}} \mathrm{TMS}^{r}$ & $130 ; 5,0$ & II & B & 4 \\
\hline & & & III & C & 2 \\
\hline & & & IV & D & 1 \\
\hline \multirow[t]{3}{*}{ S. sonnei } & $\mathrm{TMS}^{\mathrm{r}}$ & 130 & VI & $\mathrm{F}$ & 6 \\
\hline & & & VII & G & 5 \\
\hline & AMPr TMS ${ }^{r}$ & $130 ; 5,0$ & VIII & $\mathrm{H}$ & 4 \\
\hline
\end{tabular}

a AMP: ampicilina; AMS: ampicilina + sulbactama; TET: tetraciclina; CMP: cloranfenicol; TMS: trimetoprima + sulfametoxazol.

b Patrones de susceptibilidad frente a diferentes cepas de Pseudomonas aeruginosa productoras de piocinas.

c Perfiles de bandas obtenidos por amplificación de segmentos repetitivos mediante reacción en cadena de la polimerasa.

palmente en niños y otros grupos de pacientes en quienes el uso de quinolonas fluoradas no está autorizado. El fenotipo de resistencia combinada frente a AMP, CMP, TET y TMS fue también el que encontraron con mayor frecuencia Navia y colaboradores en Tanzania (13), Brito Alayón en Argentina (23) y Farfán y colaboradores en Chile (24). Dicha resistencia se vinculó con la presencia de plásmidos transferibles con pesos moleculares de $94,5 \mathrm{a} 120 \mathrm{~kb}$.
En el presente trabajo se encontró más de un piocinotipo en cepas que tenían un mismo fenotipo de resistencia a antibióticos. Esta información es de gran utilidad si se desea realizar una discriminación preliminar de los aislamientos según su origen clonal antes de determinar los genotipos, ya que la tipificación por piocinas resulta más económica y reproducible. El poder discriminatorio de la tipificación por piocinas puede llegar a 0,824 y aumen- tar a 0,945 cuando se combinan el piocinotipo y el antibiotipo (7). El poder discriminatorio es la probabilidad de determinar correctamente que dos cepas tipificadas mediante un carácter fenotípico tienen diferente origen clonal. Esta metodología se ha aplicado con éxito en varios estudios en los que se pudo tipificar a la mayoría de las cepas mediante su resistencia a las piocinas (14).

La tipificación bacteriana mediante el análisis de la dotación plasmídica de los aislamientos estudiados en el presente trabajo resultó de escasa utilidad, ya que este método permite caracterizar mejor las cepas que presentan dos plásmidos o más (25). El hallazgo de 1 a 8 plásmidos de diferentes tamaños en cepas de $S$. flexneri y de hasta 12 plásmidos en cepas de $S$. sonnei permitió relacionar la resistencia de esas cepas a los antibióticos con elementos genéticos transferibles que pueden pasar hacia otras bacterias y transmitir la resistencia $(26,27)$.

Por su parte, la metodología de REP-PCR ha sido ampliamente aplicada por diversos autores para tipificar cepas, ya sean provenientes de casos aislados o de brotes. Al igual que otras técnicas que amplifican secuencias repetitivas (28), esta ha demostrado poder discriminar cepas de diferentes orígenes con una precisión similar a la obtenida mediante electroforesis en campo pulsátil (13), que se considera la técnica patrón para la tipificación molecular de cepas. En el presente trabajo, la REP-PCR permitió demostrar que cepas con un mismo perfil de resistencia tenían diferentes patrones electroforéticos y viceversa. Esto podría indicar que, aun cuando algunas de las cepas proviniesen de un mismo clon, la presión selectiva impuesta por el uso de medicamentos antimicrobianos pudo haber llevado a la aparición de nuevos patrones de resistencia debido a la incorporación de elementos extracromosómicos transferibles. Por otro lado, la correlación encontrada entre los piocinotipos y los patrones genéticos observados mediante la REP-PCR podría indicar la conveniencia de realizar la tipificación fenotípica antes de aplicar las técnicas 
FIGURA 1. Perfiles plasmídicos representativos de las cepas de Shigella con multirresistencia. Región nordeste de Argentina, 19982002



Líneas 1, 2 y 3: Shigella flexneri; líneas 4 y 5: Shigella sonnei.

FIGURA 2. Patrones electroforéticos representativos de los diferentes grupos que se observaron en cepas de Shigella estudiadas mediante la amplificación de segmentos repetitivos de ADN con la reacción en cadena de la polimerasa. Región nordeste de Argentina, 1998-2002
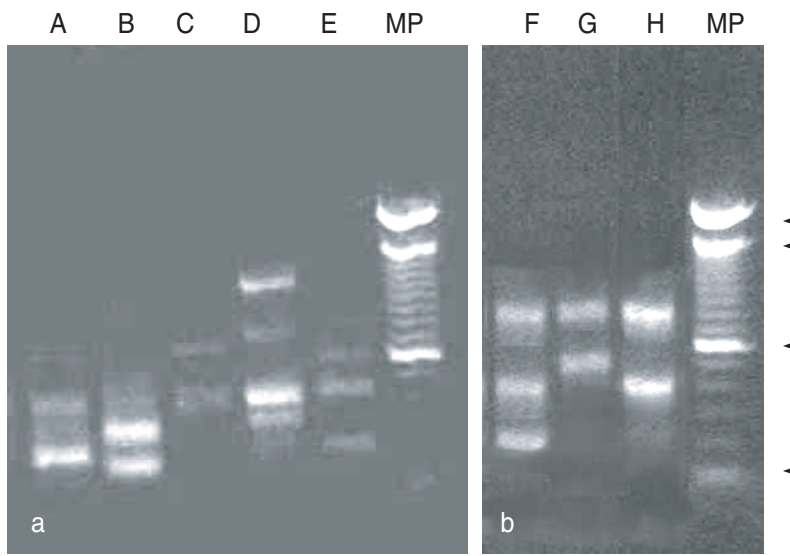

Peso molecular

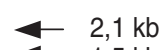

$1,5 \mathrm{~kb}$

$0,6 \mathrm{~kb}$

$0,1 \mathrm{~kb}$

a: Shigella flexneri; b: Shigella sonnei; MP: marcador de pesos moleculares de $100 \mathrm{bp}$. de tipificación genética, que resultan más costosas y no se hacen en todos los laboratorios.

El escaso número de perfiles obtenidos con los diferentes métodos utilizados puede indicar que la aplicación aislada de cualquiera de estas técnicas de tipificación puede conducir a conclusiones epidemiológicas erróneas y que solo su uso combinado en un contexto temporal y geográfico adecuado puede llevar a correlaciones epidemiológicas correctas entre diferentes aislamientos.

El análisis de los resultados obtenidos mediante la combinación de las diferentes metodologías empleadas permitió concluir, tomando en consideración las fechas y lugares en que se realizaron los aislamientos, que las cepas provenían de casos aislados y no de brotes. La única excepción fueron las seis cepas de $S$. flexneri resistentes a AMP, AMS, TET, CMP y TMS, las cuales pertenecían al piocinotipo I y un perfil de bandas del tipo A según la REP-PCR, lo cual refleja que puede existir una relación clonal y epidemiológica entre dichas cepas.

Los resultados obtenidos indican que, en las especies de Shigella estudiadas, la resistencia a los antibióticos de uso más frecuente es elevada, por lo que se deben poner en marcha actividades de vigilancia orientadas a detectar y controlar la aparición de nuevas cepas resistentes. La aplicación de técnicas de tipificación epidemiológica puede ayudar a conocer con mayor precisión la distribución y evolución de las cepas resistentes circulantes.

\section{REFERENCIAS}

1. Sack R, Rahman M, Yunus M, Khan E. Antimicrobial resistance in organisms causing diarrheal disease. Clin Infect Dis. 1997; 24(suppl 1):S102-5.

2. Suárez ME, Carvajal L, Culasso C, Paredes M. Resistencia de Shigella spp. a los antimicrobianos en Córdoba, Argentina, durante el período 1990-1997. Rev Panam Salud Publica. 2000;7(2):113-7.

3. Tompkins L. The use of molecular methods in infectious diseases. N Engl J Med. 1992; 327(18):1290-7.

4. Blanc DS, Siegrist HH. Typage bactérien: méthodes et valeur épidémiologique. Swiss-
NOSO. 1995;2(1):21-3. Hallado en: http:// www.hospvd.ch/swiss-noso/f21a3.htm. Acceso el 9 de marzo de 2004.

5. National Committee for Clinical Laboratory Standards. Performance standards for antimicrobial susceptibility tests. $6{ }^{a}$ ed. Wayne, Pennsylvania, USA: NCCLS; 1998.

6. Wahba $\mathrm{AH}$. The production and inactivation of pyocines. J Hyg. 1963;61(4):431-40.

7. Merino LA, Hreñuk GE, Alonso JM, Ronconi MC. Intérêt de l'antibiotypie et de la bacteriocinotypie pour différencier les souches de Shigella isolées en Argentine. Bull Soc Pathol Exot. 2000;93(5):307-10.
8. Kado C, Liu S. Rapid procedure for detection and isolation of large and small plasmids. J Bacteriol. 1981;145(3):1365-73.

9. Nakamura M, Sato S, Ohya T, Susuki S, Ikeda $\mathrm{S}$. Plasmid profile analysis studies of animal Salmonella typhimurium infection in Japan. J Clin Microbiol. 1986;23(2):360-5.

10. Gallardo F, Ruiz J, Towner K, Vila J. Increase in incidence of resistance to ampicillin, chloramphenicol, and trimethoprim in clinical isolates of Salmonella serotype Typhimurium with investigation of molecular epidemiology and mechanisms of resistance. J Med Microbiol. 1999;48:367-74. 
11. Rossi A, Tokumoto M, Galas M, Soloaga R, Corso A y Red Nacional de Laboratorios que participan en el Programa WHONET. Vigilancia de la resistencia a los antibacterianos en Argentina. Programa WHONET, 1995-1996. Rev Panam Salud Publica. 1999;6(4):234-41.

12. Chu Y, Houang E, Lyon D, Ling J, Ng T, Cheng A. Antimicrobial resistance in Shigella flexneri and Shigella sonnei in Hong Kong, 1986-1995. Antimicrob Agents Chemoth. 1998;42(2):440-3.

13. Navia M, Capitano L, Ruiz J, Vargas M, Urassa $\mathrm{H}$, Schellemberg $\mathrm{D}$, et al. Typing and characterization of mechanisms of resistance of Shigella spp. isolated from feces of children under 5 years of age from Ifakara, Tanzania. J Clin Microbiol. 1999;37(10):3113-7.

14. Castillo F, Carranza E, Clavel A, Rubio M, Gómez Lus R. Epidemiología de la shigelosis y colicinotipia de Shigella sonnei. Un estudio de 14 años. Enferm Infec Microbiol Clin. 1991; 9(9):530-6.

15. Litwin C, Storm A, Chipowsky S, Ryan K. Molecular epidemiology of Shigella infections: plasmid profiles, serotype correlation, and restriction endonuclease analysis. J Clin Microbiol. 1991;29(1):104-8.

16. Yadón Z, Schmunis G. Sensibilidad de Salmonella, Shigella y Vibrio cholerae a los antimicrobianos en las Américas 1940-1997. En: Salvatierra González R, Benguini Y, eds. Resistencia antimicrobiana en las Américas: magnitud del problema y su contención. Washington, D.C.: Organización Panamericana de la Salud; 2000. Pp. 24-38.

17. Prats G, Mirelis B, Llovet T, Muñoz C, Miró E, Navarro F. Antibiotic resistance trends in enteropathogenic bacteria isolated in 19851987 and 1995-1998 in Barcelona. Antimicrob Agents Chemother. 2000;44(5):1140-5.

18. Casellas JM, Casellas JM, Tome G, Pagniez G, Ivanovic S, Espinola $\mathrm{C}$, et al. Actividad in vitro de la azitromicina frente a 100 cepas de Shigella spp. aisladas de niños con diarrea aguda en Argentina y Paraguay, en comparación con otros agentes antibacterianos. Rev Panam Infect. 2000. Hallado en: http:/ /www. encolombia.com/medicina/infectologia/ infectologia4100sup-actividad.htm. Acceso el 9 de marzo de 2004.

19. Hakanen A, Kotilainen P, Jalava J, Siitonen A, Huovinen P. Detection of decreased fluoroquinolone susceptibility in Salmonellas and validation of nalidixic acid screening test. J Clin Microbiol. 1999;37(11):3572-7.

20. Vila J, Gascón J, Abadía S, Gómez J, Marco F, Moreno A, et al. Antimicrobial resistance of Shigella isolates causing traveler's diarrhea. Antimicrob Agents Chemother. 1994;38(11): 2668-70.

21. Lima A, Sidrim J, Lima N, Titlow, W, Evans M, Greenberg R. Molecular epidemiology of multiply antibiotic-resistant Shigella flexneri in Fortaleza, Brasil. J Clin Microbiol. 1997;35(5): 1061-5.

22. Boehme $\mathrm{C}$, Iglesias $\mathrm{T}$, Loyola $\mathrm{A}$, Soto $\mathrm{L}$, Rodríguez $\mathrm{G}$, Reydet $\mathrm{P}$, et al. Comparación de la susceptibilidad de especies de Shigella a antimicrobianos de uso habitual en el Hospital Regional de Temuco, Chile 1990-2001. Rev Med Chile. 2002;130(9):1021-6.

23. Brito Alayón N, Blando A, Monzón Moreno C. Antibiotic resistance patterns and plasmid profiles for Shigella spp. isolated in Cordoba,
Argentina. J Antimicrob Chemother. 1994;34: 253-9.

24. Farfán M, Flores $\mathrm{O}$, Navarro $\mathrm{N}$, Prado $\mathrm{V}$, Mora G, Toro C. Caracterización molecular de mecanismos de resistencia a cloranfenicol en cepas de Shigella flexneri aisladas en niños chilenos con diarrea aguda. Rev Med Chile. 2002; 130(3):275-80.

25. Marco F, Jiménez de Anta M. Métodos de tipificación: análisis de plásmidos. Ventajas e inconvenientes. Enferm Infec Microbiol Clin. 1993;11(2):97-101.

26. Brian MJ, Van R, Townsend I, Murray BE, Cleary TG, Pickering LK. Evaluation of the molecular epidemiology of an outbreak of multiple resistant Shigella sonnei in a day-care center by using pulsed-field gel electrophoresis and plasmid DNA analysis. J Clin Microbiol. 1993;31(8):2152-6

27. Talukder KA, Islam MA, Dutta DK, Hassan F, Safa A, Nair GB, et al. Phenotypic and genotypic characterization of serologically atypical strains of Shigella flexneri type 4 isolated in Dhaka, Bangladesh. J Clin Microbiol. 2002; 40(7):2490-7.

28. Liu PYK, Lau YJ, Hu BS, Shyr JM, Shi ZY, Tsai WS, et al. Analysis of clonal relationships among isolates of Shigella sonnei by different molecular typing methods. J Clin Microbiol. 1995;33(7):1779-83.

Manuscrito recibido el 22 de julio de 2003. Aceptado para publicación, tras revisión, el 1 de marzo de 2004.
ABSTRACT

Antibiotic resistance and molecular epidemiology of Shigella spp. in northeastern Argentina
Objectives. To evaluate the antibiotic resistance of strains of Shigella spp. isolated from feces samples from northeastern Argentina and to characterize the strains in terms of their molecular epidemiology.

Methods. We studied 132 isolates of Shigella spp. obtained from feces samples from 132 patients with diarrhea who were seen at various private and public laboratories in the Argentine provinces of Chaco and Corrientes during the period of 1998 to 2002. Each strain was characterized according to its serotype, its resistance to 13 individual or combination antibiotics, and its sensitivity to pyocins. With 52 strains selected in relation to their antimicrobial susceptibility profiles we conducted plasmid profile analysis using alkaline lysis, and the repetitive extragenic palindromic sequences were determined by amplifying repetitive DNA segments using polymerase chain reaction. The chi-square test was used to compare proportions, with a level of statistical significance of 0.05 .

Results. Shigella flexneri was the most common species $(78 \%)$, followed by S. sonnei $(22 \%)$. In general, the resistance of $S$. flexneri to the antibiotics studied was greater than that of $S$. sonnei, and this difference was statistically significant $(P<0.001)$ for ampicillin, tetracycline, chloramphenicol, and the combination of ampicillin and sulbactam. The $S$. flexneri strains also showed multiple resistance more often than $S$. sonnei strains $(84.5 \%$ vs. $31.0 \% ; P<0.001)$. The strains isolated from S. flexneri were grouped into five pyocin types, three plasmid profiles, and five patterns of repetitive palindromic sequences. The strains of $S$. sonnei formed three pyocin types, two plasmid profiles, and three patterns of repetitive palindromic sequences.

Conclusions. Given that the Shigella species that were studied showed a high level of resistance to the most frequently used antibiotics, surveillance activities should be implemented in order to detect and control the appearance of new resistant strains. Applying epidemiological typing techniques can provide more precise information about the distribution and evolution of resistant strains of circulating microorganisms. 